\title{
Erratum to: The Development of the Agrifood Market in China: Opportunities and Challenges
}

\author{
Mian Wu
}

Erratum to:

China, New Zealand, and the Complexities of Globalization

DOI 10.1057/978-1-137-51690-9_6

Chapter 6 was misattributed to multiple authors, but was corrected.

The updated original online version of the chapter can be found under DOI 10.1057/978-1-137-51690-9_6

\section{Wu (凶)}

School of Management, Massey University School of Management, Auckland, New Zealand 